\title{
PERFUSION MODEL APPROXIMATION IN CEREBRAL TUMOURS: FEATURE IMAGES AND COMPUTATION TIMES
}

\author{
Kai Ritschel ${ }^{1}$, Ioannis Pechlivanis ${ }^{2}$, Christian Kensy ${ }^{1}$, Rasmus Risse ${ }^{1}$ and Susanne Winter ${ }^{1}$ \\ ${ }^{1}$ Institute for Neural Computation, Ruhr Universität Bochum, Germany \\ ${ }^{2}$ Neurologische Universitätsklinik Mannheim, Mannheim, Germany \\ Kai.Ritschel@ini.Ruhr-Uni-Bochum.de
}

\begin{abstract}
Ultrasound contrast imaging can be used to visualize tumours due to changes in the local perfusion. Quantitative analysis of the data may establish features usable for classification of the scanned tissue. In this work we approximate model functions for the contrast agent concentration time course of every pixel, while reducing computation times by means of parallel computing, enabeling generation of feature images on the basis of model parameters in acceptable times for a clinical application. Keywords:
\end{abstract}

Perfusion Imaging, Tumour Imaging, Feature Imaging, Ultrasound Contrast Imaging, Model Function

\section{Introduction}

The use of ultrasound contrast agents allows for perfusion specific imaging. Contrast enhanced ultrasound imaging (CEUS) is e.g. applied in diagnosis of ischemic stroke or neoplasia. Neoplasia induce growth of new morphological differentiated blood vessels in their vicinity and thus are identifiable, for example in liver tissue [1] or in cerebral tumours [2]. To assess the perfusion of tissue, a bolus of contrast agent is injected and scanned while passing an area. The interpretation of this video data can be complex and is typically performed by visual parsing. Model functions were introduced to allow for a quantitative interpretation by fitting the model parameters to the time course of the contrast agent concentration [3]. As recently demonstrated for thyroid nodules [4], the parameters of model functions can be used to differentiate tumour tissues. In a previous work we presented a more flexible and precise model to fit the data, including a possible second peak [5]. The use of more parameters and possible features of the image data may enable an automated classification based on a quantitative analysis of CEUS Data. However, feature computation for video data and model function approximation are computational intensive processes. A clinical application will only be viable within acceptable computation times. In this study we evaluated the capability of parallel computation in the given scenario to reduce computation times for application in clinical trials.

\section{Methods}

\section{Contrast Enhanced Ultrasound Data}

A total of 10 intracranial recordings were acquired in tumour resection surgery (seven globlastoma, one b-cell lym- phoma and two astrocytoma II) by applying a $2 \mathrm{ml}$ bolus of the contrast agent SonoVue (Bracco Imaging, Germany). The ultrasound system Aplio XG 500 (Toshiba Medical Systems, Japan) was used for the acquiration of a 2D-video with a resolution of $408 \times 340$ pixels and approximately 500 single frames.

\section{Model Functions and Approximation Method}

The bolus injection of the contrast agent leads to a fast rise in the concentration followed by a wash-out phase. The gamma variate function model (Eq.1) [3] was applied to describe the time course of the concentration agent in every pixel of the video data.

$$
I_{\mathrm{var}}(t)=g_{\mathrm{base}}+g_{\mathrm{peak}} \cdot\left(\frac{t+s}{t_{\mathrm{ttp}}}\right)^{\beta \cdot t_{\mathrm{ttp}}} \cdot e^{-\beta\left(t+s-t_{\mathrm{ttp}}\right)}
$$

The model parameter $g_{\text {peak }}$ describes the maximum concentration, $g_{\text {base }}$ depicts the concentration before and after the bolus passes the tissue. The parameters $s$ and $t_{\mathrm{ttp}}$ represent the start of the rising and the time to peak concentration, while $\beta$ determines the slope of the curve. To approximate the model function to fit a time course, optimal parameters have to be found. Therefore an error function was formulated as the misalignment of the model by means of euclidean distance between the data and the model. By minimizing the value of the error function an optimal set of parameters could be identified. The optimization was terminated after 300 search steps, to simulate a worst case scenario.

\section{Feature Images}

For a qualitative interpretation of the video data, features, such as the model function parameters, were visualized as feature images. Some features can be assessed visually and support the surgeon to evaluate the tissue perfusion. Some information about the concentration time course of the contrast agent used in the assessment of perfusion are directly represented by parameters of the model function such as the amplitude $g_{\text {peak }}$ or the time needed to reach the peak $t_{\mathrm{ttp}}$. In this work the parameters $g_{\text {peak }}$ (maximum amplitude), $g_{\text {base }}$ (base concentration level) and $\beta$ (slope) of the gamma variate model function were depicted. 


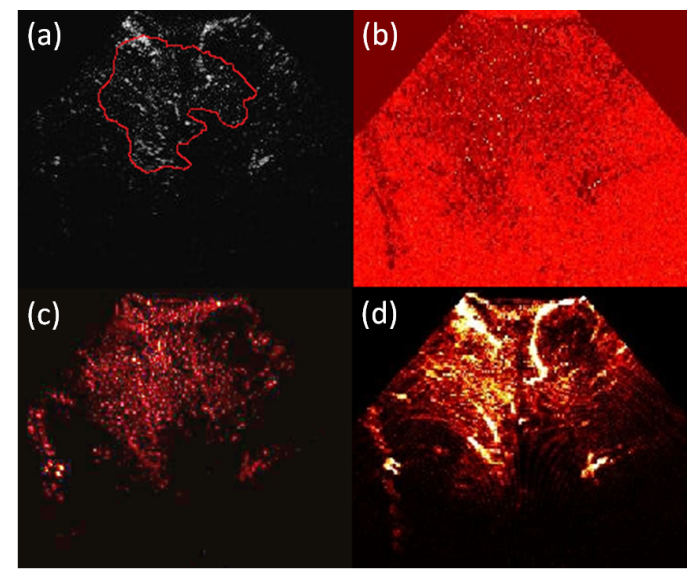

Figure 1: Orignal frame with marked tumor (a) and feature images of the parameters $\beta$ (b), $g_{\text {base }}$ (c) and $g_{\text {peak }}(\mathrm{d})$.

Table 1: Mean computation times and std. deviation in seconds for 1, 2, 8, 12 and 16 parallel threads. Additionally the computation times on an undersampled data set are shown.

\begin{tabular}{lrrrrr} 
\# of Threads: & 1 & 2 & 8 & 12 & 16 \\
\hline Mean Time & 4333 & 2249 & 856 & 1084 & 849 \\
Std deviation & 14.5 & 14.5 & 10.9 & 16.3 & 2.1 \\
\hline Undersampled & 1058 & 558 & 209 & 271 & 209 \\
\hline
\end{tabular}

\section{Multithreading}

To reduce computation times the architecture of a software system was adapted to support CPUs with multiple cores. The time course of every pixel can be computed independently and thus calculated in parallel. For the implementation the boost thread library was used to flexibly fit any given number of CPU cores. The time measurements were taken on a Intel Core I7 with 4 cores and up to 8 threads in parallel due to the hyper threading technology.

\section{Results}

\section{Feature Images}

Figure 1 depicts an original frame (a) taken at peak concentration and three parameter maps generated according to $\beta$ (b), $g_{\text {base }}$ (c) and $g_{\text {peak }}(\mathrm{d})$ of the approximated model. The area of the tumour is marked in red. While a differentiation of tumour tissue is difficult, the calculated features allow for an additional perspective. The feature image of $g_{\text {peak }}$ showed increased values in the area of larger blood vessels, the parameter $g_{\text {base }}$ consistently depicted the perfused tissue. The slope $\beta$ of the modeled concentration curve showed increased values in an even larger area, including the tumour.

\section{Computation Times}

Ten data sets were approximated with the gamma variate model function in full resolution with 1, 2, 8, 12 and 16 parallel threads. Using one thread took $4333 \mathrm{sec}$ for one video, the use of two parallel threads cut the time almost in half. This effect continued to the maximum number of parallel threads and reduced total computation time to 856 sec. Assigning 16 threads to a eight core CPU again improved the computation time significantly to $849 \mathrm{sec}$ (sign test, $p<0.05$ ). With the exception of 12 parallel threads the computation time decreased when using more threads. One dataset was sampled down to half of the original resolution and a model function was fitted to the new time courses. The computation times were cut by four in all cases, leading to a final computation time of $209 \mathrm{sec}$ with 8 and 16 parallel threads.

\section{Discussion}

The large expense of computation time can be reduced by using parallel computing. This allows for the use of high resolution data to qualitatively assess the tissue perfusion via ultrasound contrast agent dynamics. It was shown, that the combination of minor reduction of the resolution and parallel computing leads to acceptable computation times (less than four minutes) for a clinical setting, without losing much information. The calculated features may also be applicable for an automated classification of the perfusion data.

The increased computation times for 12 parallel threads can be explained by interference in the allocation of processing time by the operating system. However no explanation for the significant decrease in computation times between 8 and 16 threads was found.

Furthermore, the number of update steps may be largely reduced by using a stop criterion based on the convergence of the algorithm. In addition to the model function parameters other features will be defined and evaluated for their usability in a classification scenario, leading towards a quantitative analysis of ultrasound perfusion imaging data.

\section{Bibliography}

[1] T. Albrechtand et al., "Imaging of liver metastases with contrast-specific low-MI real-time ultrasound and SonoVue," EUR RADIOL, vol. 13, no. 3, pp. 79-86, 2003.

[2] M. Engelhardt et al., "Intraoperative contrast enhanced perfusion imaging of cerebral tumors," in IEEE Ultrasonics Symposium, vol. 1, pp. 743-746, 2005.

[3] M. T. Madsen, "A simplified formulation of the gamma variate function,” PHYS MED BIOL, vol. 37, 1992.

[4] U. Nemec et al., "Quantitative evaluation of contrastenhanced ultrasound [...] for differentiation of benign and malignant thyroid nodules[...]," EUR RADIOL, 2012.

[5] K. Ritschel, C. Dekomien, and S. Winter, "Modellfunktion zur Approximation von Ultraschallkontrastmittelkonzentration zur semi-quantitativen Gewebeperfusionsbestimmung," Bildverarbeitung für Medizin, pp. 159-164, 2012. 